\title{
Acute Bilateral Renal Vein Thrombosis Secondary to Sepsis from Pyelonephritis
}

\author{
Leslie A. Harris, Marvin J. Van Every, Larry J. Fundell \\ Department of Medical Education (LAH), Gundersen Lutheran Medical Foundation, Department of \\ Urology (MJvE) and Department of Diagnostic Radiology (LJF), Gundersen Lutheran Health System, USA
}

A 62-year-old obese female with no known chronic medical problems presented to the emergency department complaining of increasing fatigue and generalized weakness over several weeks duration. She appeared toxic with tachycardia, tachypnea, and hypotension. Initial laboratory tests revealed white blood cell count of $23,000 / \mu \mathrm{L}$ with a left shift, sodium of $151 \mathrm{mmol} / \mathrm{L}$, potassium of $5.3 \mathrm{mmol} / \mathrm{L}$, bicarbonate of $6 \mathrm{mmol} / \mathrm{L}$, chloride of $121 \mathrm{mmol} / \mathrm{L}$, creatinine of $1.8 \mathrm{mg} / \mathrm{dL}$, glucose of $700 \mathrm{mg} / \mathrm{dL}$, and positive serum ketones. Her urine was grossly bloody, and urinalysis revealed greater than 100 white blood cells and 100 bacteria per high-power field. Due to impending respi- ratory failure, she was intubated and transferred to the intensive care unit with a diagnosis of diabetic hyperosmolar non-ketotic acidosis and sepsis from pyelonephritis.

On examination, the patient was noted to grimace with abdominal palpation. Bedside abdominal ultrasound was obtained and suggested a left renal mass, but imaging was suboptimal. After stabilization with intravenous (IV) fluids, insulin, and antibiotics, a computed tomography (CT) scan of abdomen and pelvis with IV contrast was ordered and showed bilateral RVTs and a $7.3 \times 4 \times$ $4 \mathrm{~cm}$ heterogeneously enhancing solid mass with cystic components of the left kidney (Figure-1).

Figure 1 - Initial CT scan showing perfusion changes consistent with bilateral renal vein thrombosis and a heterogeneously enhancing solid mass with cystic components of left kidney.
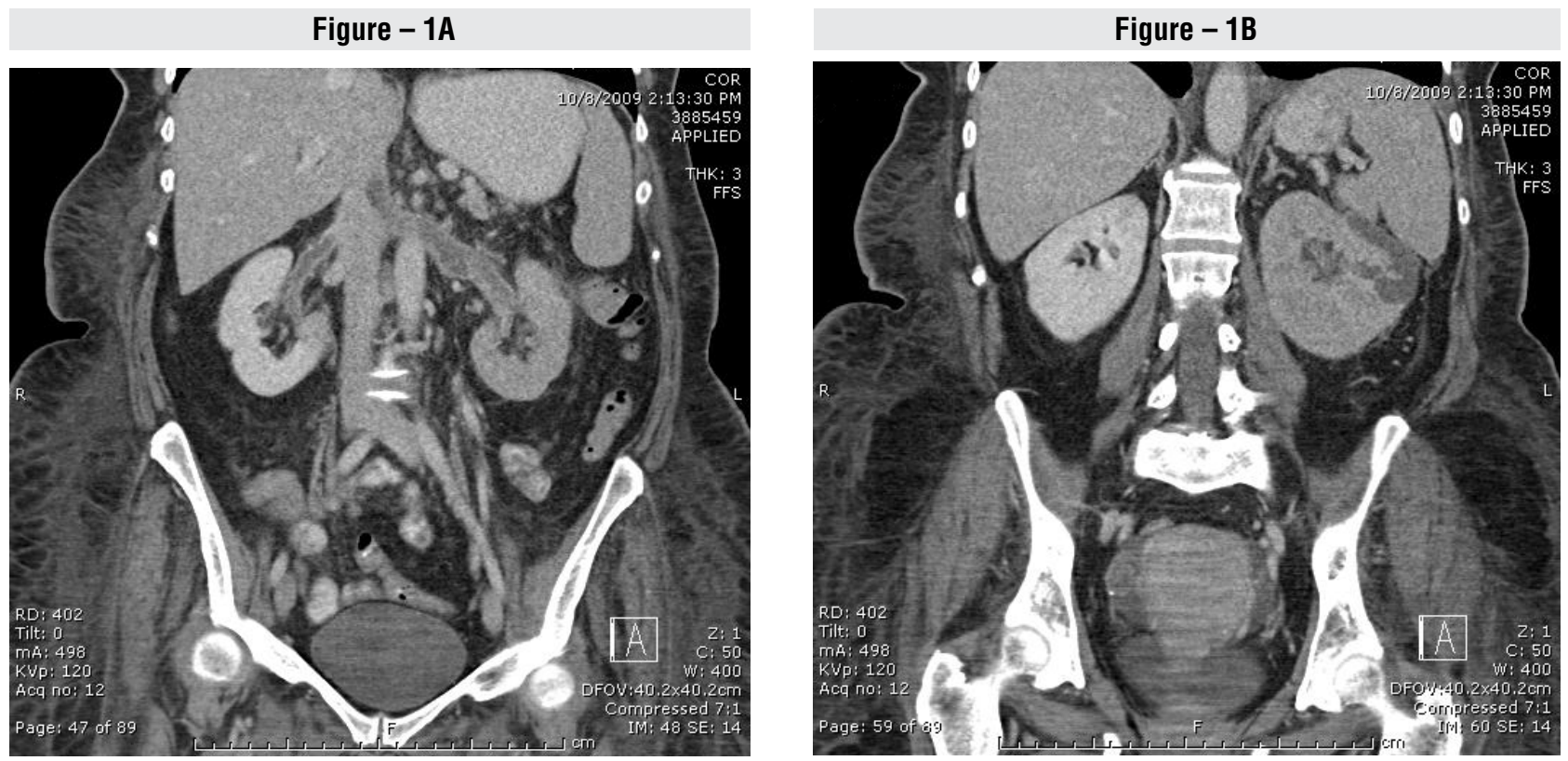
Several lung nodules were also evident. The radiographic findings were most consistent with pyelonephritis, bilateral septic thrombosis, and septic pulmonary emboli. Anticoagulation was initiated with IV heparin and warfarin. Heparin was discontinued when the International Normalized Ratio (INR) was therapeutic. Pyelonephritis was treated with 3 weeks of ciprofloxacin after urine cultures grew Klebsiella pneumonia. The patient did well and was discharged from the hospital after 10 days.

A follow-up CT scan at 4 weeks demonstrated improvement of the pyelonephritic changes and resolution of the right RVT. After 20 weeks of treatment, her creatinine improved to 1.0 , and her CT scan showed resolution of both RVTs (Figure-2). Warfarin was discontinued, and the patient continued to do well in follow-up.

RVT is most often seen in the pediatric population but has been reported in adults with variable presentations. Acute RVT classically presents as macroscopic hematuria and flank pain. This pain originates from renal vein obstruction and absent collateral venous return (1).
Causes of RVT are nearly all due to hypercoaguable states. The most common cause of RVT is nephrotic syndrome. Other etiologies include renal cell carcinoma, renal transplantation, antiphospholipid antibody syndrome, lupus, trauma, oral contraceptive use, and sepsis (2).

Doppler ultrasonography is a good screening test for RVT and will show decreased flow in the vein or a discrete mass. Additionally, RVT can be diagnosed with CT by demonstrating thrombus in the renal vein and nephromegaly. CT scan can also show ipsilateral prolonged corticomedullary differentiation, thickening of the renal fascia, stranding of the perinephric fat, and perinephric hemorrhage, which are suggestive of RVT (1).

Treatment of RVT is important, as high morbidity and mortality rates have been reported if RVT is left untreated (3). In the past, the mainstay of treatment was surgery, which generally included a nephrectomy. Surgery is now rarely required because conservative management, including supportive therapy, anticoagulation, and thrombolysis, are generally very effective. Thrombolytics are generally reserved for patients with

Figure 2 - Follow-up CT scan at 20 weeks showing resolution of bilateral renal vein thrombosis.
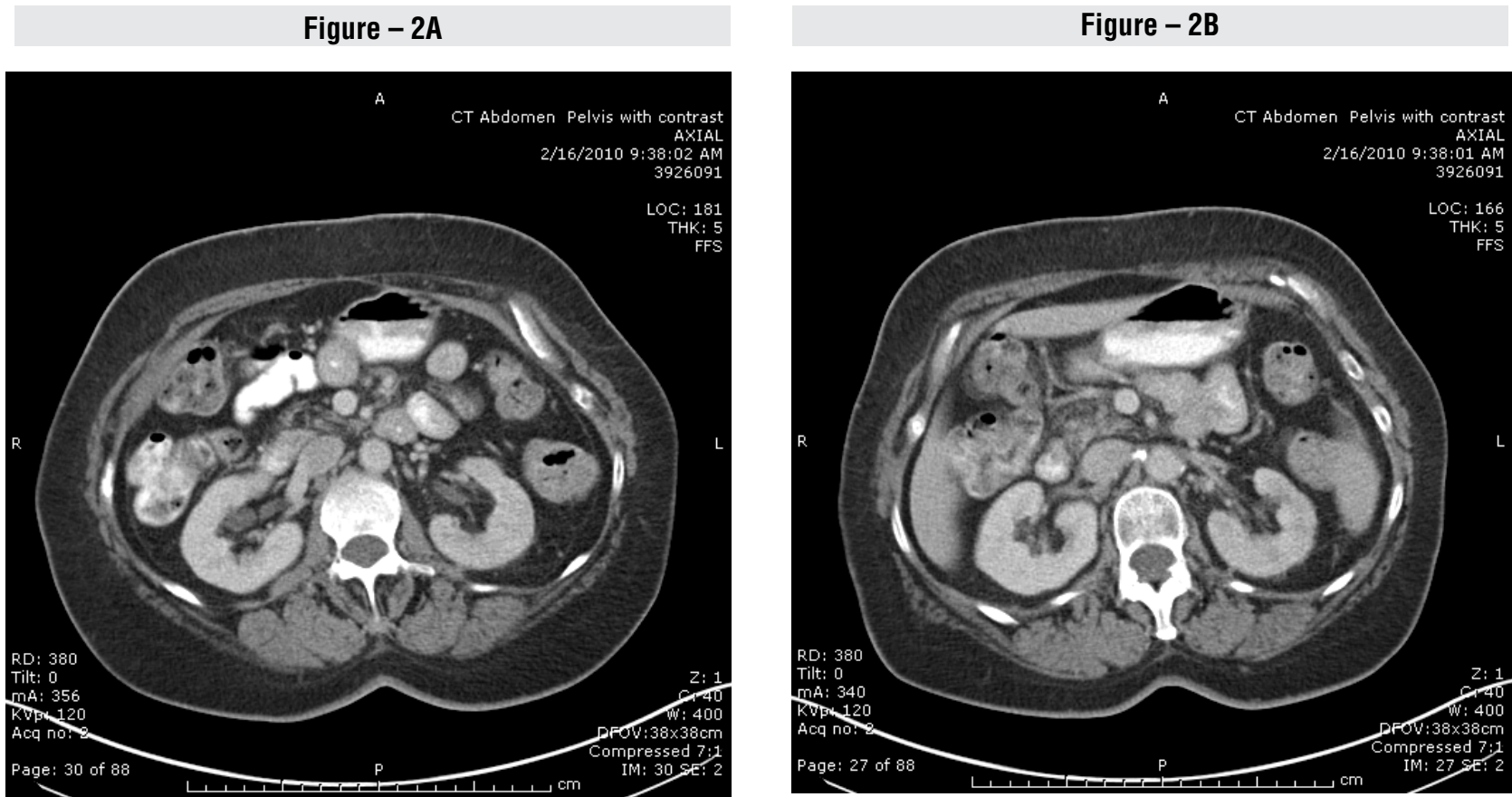
severe disease, poor prognosis, massive clot burden, and bilateral RVT with acute renal failure (1). In summary, RVT can manifest as hematuria and loin pain and is generally caused by a hy-

\section{REFERENCES}

1. Witz M, Kantarovsky A, Morag B, Shifrin EG: Renal vein occlusion: a review. J Urol. 1996; 155: 1173-9.

2. Qian $Q$, Saucier NA, King BF: Acute bilateral renal vein thrombosis. Am J Kidney Dis. 2009; 54: 975-8. percoaguable state. Diagnosis is made with clinical suspicion and imaging. Treatment depends on the patient and, as our case demonstrates, conservative management can lead to an optimal outcome.

3. Wysokinski WE, Gosk-Bierska I, Greene EL, Grill D, Wiste $\mathrm{H}$, McBane RD 2nd: Clinical characteristics and long-term follow-up of patients with renal vein thrombosis. Am J Kidney Dis. 2008; 51: 224-32.
Correspondence address: Marvin J. Van Every, MD Mail Stop C04-004 Department of Urology Gundersen Lutheran Health System 1900 South Avenue La Crosse, WI 54601 FAX: + 16087751565 E-mail: mjvaneve@gundluth.org 